\title{
Mixing of the vibrational angular momentum components of multiply degenerate vibronic states of benzene by vibrational $l$-type resonance
}

\author{
Eberhard Riedle \\ Institut für Physikalische und Theoretische Chemie, Technische Universität München, Lichtenbergstr. 4, \\ W-8046 Garching, Germany
}

and

Josef Pliva

Department of Physics, The Pennsylvania State University, University Park, PA 16802, USA

Received 17 September 1990

\begin{abstract}
The rotationally resolved spectra of the $6_{0}^{1} 10_{0}^{2}$ and $66_{0}^{1} 16_{0}^{2}$ vibronic transitions of benzene at low rotational temperature are reported and analyzed in detail. Deperturbed spectroscopic constants for the $6^{1} 10^{2}$ and $6^{1} 16^{2}$ states are reported which reproduce the observed line positions to within experimental accuracy. The splitting of $17.9 \mathrm{~cm}^{-1}$ between the two subbands of the $60_{0}^{1} 10_{0}^{2}$ transition and of $6.2 \mathrm{~cm}^{-1}$ for the $6_{0}^{1} 16_{0}^{2}$ transition is found to be due to vibrational l-type resonances with matrix elements of 8.91 and $2.65 \mathrm{~cm}^{-1}$, respectively. These large resonances cause strong distortions of the rotational structure and mix the vibrational angular momentum substates $\nu_{6}^{\mp}+2 \nu_{10}^{0}$ and $\nu_{6}^{ \pm}+2 \nu_{10}^{ \pm 2}$ nearly completely and the substates $\nu_{6}^{\mp}+2 \nu_{16}^{0}$ and $\nu_{6}^{ \pm}+2 \nu_{16}^{\mp 2}$ substantially. The importance of the mixing for the intramolecular vibrational redistribution (IVR) and the decay behaviour of $S_{1}$ benzene is discussed.
\end{abstract}

\section{Introduction}

Recent improvements in experimental techniques have allowed the observation of IR [1-4], Raman $[5,6]$ and UV spectra [7-14] of the prototype organic molecule benzene with rotational resolution. These spectra have been analyzed in great detail and a great number of spectroscopic constants were determined with unprecedented precision for both the $S_{0}$ electronic ground state and the $S_{1}$ excited state. So far analysis was restricted to fundamental bands in the IR and Raman spectra and fundamental bands and some progressions in the totally symmetric $\nu_{1}$ mode in the electronic spectrum, as these transitions are by far the strongest ones. Information on the wealth of "background" states, i.e. all the overtones and combination states, was only deduced through the observed perturbations in the spectra.

These background states are, however, responsible for the intramolecular vibrational redistribution (IVR) in the molecule and ultimately for the dynamics. The spectroscopic perturbations caused by anharmonic resonance and Coriolis coupling are just the frequency domain analogue of the time domain behaviour. It is therefore most important to understand the character and couplings of the background states, which typically consist of a fair number of excited vibrational quanta. In addition, these are often multiply degenerate states, since most of the low-frequency modes of benzene are themselves degenerate.

Contrary to the IR spectrum, the UV spectrum displays transitions to some of these combination states with sufficient intensity for experimental observation. Two of the most prominent transitions are the $6_{0}^{1} 10_{0}^{2}$ and the $6_{0}^{1} 16_{0}^{2}$ bands. Both lead to sixfold degenerate rovibronic states differing in vibrational an. gular momentum and a strong first order HerzbergTeller induced band plus a much weaker third order 
band is expected to be seen in each case. The expected rotational structure of the bands was determined in the early work of Callomon, Dunn and Mills [15], but no agreement with the observed spectrum was found and hence the vibrational assignment remained uncertain. More detailed studies by Atkinson and Parmenter confirmed these difficulties [16].

A secure vibrational assignment was later reached by Stephenson, Radloff and Rice through the observation of single vibronic level emission spectra in a jet [17]. They found that both symmetry allowed components of the $6_{0}^{1} 10_{0}^{2}$ transition are seen in the fluorescence excitation spectrum with a surprisingly large splitting of $18 \mathrm{~cm}^{-1}$ and a nearly even intensity ratio of 3.0 to 2.7 . Similarly, for the $6_{0}^{1} 16_{0}^{2}$ transition a splitting of $6 \mathrm{~cm}^{-1}$ and a ratio of 2.9 to 1.2 was observed. The splittings could only be explained by large anharmonic constants, the intensity ratios remained unclear, and no explanation could be given for the previously observed rotational contours. In addition, in later work Stephenson and Rice reported that excitation through the higher-energy component of each transition results in a slower fluorescence decay than excitation through the lower component [18]. This was explained through selective coupling of the different vibrational momentum components to other $S_{1}$ states [19].

All the studies mentioned above were Doppler limited at best and therefore a detailed rotational analysis could not aid the understanding of the complicated situation. We will now present sub-Doppler jet spectra of the $6_{0}^{1} 10_{0}^{2}$ and $6_{0}^{1} 16_{0}^{2}$ transitions with virtually completely resolved rotational structure. A detailed analysis is performed which shows that the different vibrational angular momentum components are strongly coupled and mixed by vibrational $l$-type resonances. In support, the spectra of the $6_{0}^{1}, 10_{0}^{2}$ and $16_{0}^{2}$ bands are presented. These transitions lead to the component states of the combination levels investigated and we can therefore obtain precise values of all constants needed for a complete modelling. Finally, the consequences of the observed strong mixing of vibrational angular momentum substates will be discussed.

\section{Measurements and evaluation of the spectra}

To obtain rotationally resolved spectra of the various electronic bands of interest, sub-Doppler resolution is needed. In previous work one of us was able to show that this is possible through the combination of an extremely narrow-band tunable UV laser with a well collimated pulsed beam of benzene seeded in $\operatorname{Ar}[11$ ]. The UV laser is a frequency-doubled pulsedamplified single mode $\mathrm{cw}$ dye laser. At a frequency width of $100 \mathrm{MHz}\left(0.0033 \mathrm{~cm}^{-1}\right)$ in the UV up to $400 \mu \mathrm{J}$ of light are available. The skimmed jet apparatus is of standard design. Details of the setup were described previously [11].

Excitation of the benzene molecules is either monitored by observation of the integrated UV fluorescence [ 11 ] to yield fluorescence excitation spectra or by ionization with the light of a second UV laser of fixed wavelength and a slight time delay to yield twophoton ionization spectra [20]. For low vibrational excess energy in the $S_{1}$ state the two methods produce identical spectra. In both cases the residual Doppler width in the jet and the line width of the laser add to an observed width of individual rovibronic lines of $130 \mathrm{MHz}\left(0.0043 \mathrm{~cm}^{-1}\right)$. For precise relative frequency scaling of the spectra the transmission spectrum of a stabilized Fabry-Perot interferometer (FPI) of $150 \mathrm{MHz}$ free spectral range is recorded simultaneously with the molecular spectrum. For absolute calibration the well known $I_{2}$ absorption spectrum [21] is recorded (with a correction of -0.0056 $\mathrm{cm}^{-1}$ according to ref. [22]).

It was previously found that already an intensity of $10^{4} \mathrm{~W} / \mathrm{cm}^{2}$ is sufficient to partially saturate the strongest transitions in the benzene spectrum [11]. Therefore, only a small fraction of the available excitation light is used for the recording of a strong band like the $6_{0}^{1}$ band. All the other bands investigated in this work are much weaker, but they possess the same ground state levels and we can hence obtain the same amount of experimental signal by using a larger fraction of the laser light. In this way the quality of the spectra is nearly independent of the strength of the transition. The eventual limit of sensitivity in fluorescence detection is given by the laser stray light, but for the long lived states observed electronic gating can be used to differentiate between the molecular fluorescence and the stray light. In ionization detection 
too high a light intensity would allow ionization by the first laser and consequently broadening of the lines, but this was not found to be a problem for the reported bands.

Due to the limited scan range of the cw laser, a number of overlapping scans had to be recorded to obtain a complete spectrum of a particular band even at the rotational temperature of less than $10 \mathrm{~K} \mathrm{ob}-$ tained in the jet expansion. Data were carefully merged on a VAX 8200 computer and digitally smoothed by a polynomial filter [23]. The position of the strong lines was determined with an algorithm given by Snyder [24]. Weak features were evaluated manually in an interactive graphical computer program. A complete list of line positions and assignments can be obtained from the authors upon request.

After assignment of the spectra, the spectroscopic constants were extracted with the aid of the computer program SYMTOP [25]. For the $\mathrm{S}_{0}$ electronic ground state the value of $B_{0}^{\prime \prime}$ and the quartic centrifugal distortion constants given by Pliva and Pine [1] were used. The value of $C_{0}^{\prime \prime}$ was calculated from $B_{0}^{\prime \prime}$ and the extrapolated inertial defect of benzene as reported by Oldani et al. [26]. All ground state constants are summarized in table 1 . We found that the centrifugal distortion constants of the various excited states could not be determined significantly from the available spectra and the changes upon electronic excitation were therefore constrained to zero throughout.

\section{Analysis of the spectra}

Before we start with the analysis of the various bands observed, we want to summarize some of the notations used. This seems necessary, since different schemes are currently used in the IR and UV spectroscopy of benzene.

Table 1

Ground state constants of $\mathrm{C}_{6} \mathrm{H}_{6}\left(\mathrm{~cm}^{-1}\right)$. All values from ref. [1], except $C_{0}$ which was calculated according to ref. [26]

\begin{tabular}{ll}
\hline$B_{0}$ & 0.1897618 \\
$C_{0}$ & 0.0948577 \\
$D_{J}$ & $3.934 \times 10^{-8}$ \\
$D_{J K}$ & $-6.90 \times 10^{-8}$ \\
$D_{K}$ & $3.21 \times 10^{-8}$ \\
\hline
\end{tabular}

The $\mathrm{E}_{2 \mathrm{~g}}$ mode $\nu_{6}$, the $\mathrm{E}_{1 \mathrm{~g}}$ mode $\nu_{10}$ and the $\mathrm{E}_{2 \mathrm{u}}$ mode $\nu_{16}$ in the Wilson numbering [27] correspond to $\nu_{18}$, $\nu_{11}$ and $\nu_{20}$, respectively, in Herzberg's numbering [28].

The nomenclature for vibronic transitions in benzene is normally given by $X_{n}^{m}$, where $m$ is the number of quanta of vibration $X$ present in the ${ }^{1} B_{2 u} S_{1}$ state and $n$ is the number of quanta in the ${ }^{1} \mathrm{~A}_{1 \mathrm{~g}} \mathrm{~S}_{0}$ state. All modes for which $m$ or $n$ is not equal to zero are written in succession. Labels such as $X^{m}$ and $X_{n}$ refer to specific vibronic levels in the excited and ground states, respectively.

For degenerate modes the value of the vibrational angular momentum quantum number $l$ is added to give a complete description for a transition like the $6_{0}^{1} l_{0}^{ \pm} 16_{0}^{2} l_{0}^{\mp 2}$ band or the upper state of this transition, the $6^{1} l^{ \pm} 16^{2} l^{\mp 2}$ state. The upper signs apply to the $(+l)$ component of the degenerate state that receives the $\Delta K=+1$ transitions in the perpendicular bands observed exclusively in the vibronic spectrum and the lower signs apply to the $(-l)$ component receiving the $\Delta K=-1$ transitions.

Since all bands studied in this work originate from the vibronic ground state of the molecule, we do not have to label $S_{0}$ states and we can use the notation more common in infrared spectroscopy for labeling of the excited states. The abovementioned vibronic state is therefore denoted by $\nu_{6}^{ \pm}+2 \nu_{16}^{\mp 2}$. It is implicitly assumed that all discussed states are within the $S_{1}$ electronic state. For the same reason, also the usual prime is omitted in the description of most excited state spectroscopic constants.

\subsection{Transitions to the component states}

Due to the symmetries of the $\nu_{6}, \nu_{10}$ and $\nu_{16}$ states not only the transitions to the combination states $\nu_{6}+2 \nu_{10}$ and $\nu_{6}+2 \nu_{16}$ are symmetry allowed but also the transitions to the components $\nu_{6}, 2 \nu_{10}$ and $2 \nu_{16}$. The latter ones will be discussed first to obtain precise reference values of the various spectroscopic constants.

\subsubsection{The $6_{0}^{I}$ band}

A Doppler-limited room temperature spectrum of the $6_{0}^{1} l_{0}^{\mp}$ band displaying a characteristic rotational contour was analyzed by Callomon, Dunn and Mills $(C D M)$ in 1966 [15]. These authors developed most 
of the theoretical framework for the understanding of the electronic spectrum of benzene, but the limited resolution of their spectrum did not allow the precise determination of the spectroscopic constants. This was only possible, when the sub-Doppler spectrum of the band became available [11]. The analysis was performed according to the energy formula

$$
\begin{aligned}
& \nu_{6}^{\mp}(J, K)=\nu_{0}\left(\nu_{6}^{\mp}\right)+\Delta B\left[J(J+1)-K^{2}\right] \\
& \quad+\Delta C K^{2} \pm 2 K\left(C \zeta_{6}\right) .
\end{aligned}
$$

The sign of the Coriolis term was chosen according to the recipe given by Hoy and Mills [29].

We now include the well known rotational $l$-type resonance [29],

$$
\begin{aligned}
& \left\langle\nu_{6}^{-}(J, K+1)\left|h_{2}^{+}\right| \nu_{6}^{+}(J, K-1)\right\rangle \\
& =\frac{1}{2} q_{6}^{-} F(J, K) F(J, K+1),
\end{aligned}
$$

in the reanalysis of the same experimental spectrum, where

$F(J, K)=[J(J+1)-K(K-1)]^{1 / 2}$.
Thus a simple $2 \times 2$ Hamiltonian matrix was used for the treatment of the upper state of the $6_{0}^{1} l_{0}^{\mp}$ band, with the diagonal elements given by eq. (1) and the off-diagonal elements given by eq. (2). All assignments remained unchanged, but a somewhat better fit resulted. The constants of the $\nu_{6}^{\mp}$ state obtained in this way are summarized in table 2.

As can be expected, no large changes occurred due to the more detailed description. The slight change in the value of $C_{6}^{\prime}$ is mainly due to the value of $C_{0}^{\prime \prime}$ adopted (compare section 2 and table 1 ). We do, however, obtain a value for $q_{\overline{6}}^{-}$.

\subsubsection{The $10_{0}^{2}$ band}

The $2 \nu_{10}$ state is triply degenerate. The $2 \nu_{10}^{0}$ substate of $b_{2 u}$ symmetry (including the symmetry of the $S_{1}$ electronic state) does not give rise to an electronic transition, while the $\mathrm{e}_{1 \mathrm{u}}$ substate $2 \nu_{10}^{\mp 2}$ gives rise to the $10_{0}^{2} l_{0}^{2}$ band. The experimental spectrum of this band is shown in fig. 1 (upper trace). The sub-Doppler resolution allows the separation of most rovibronic lines. The overall appearance of the band is quite different from the $6_{0}^{1} l_{0}^{\mp}$ band. This is due to the

Table 2

\begin{tabular}{|c|c|c|c|c|c|}
\hline & $\nu_{6}^{\mp}$ & $2 \nu_{10}^{\mp 2}$ & $2 \nu_{16}^{ \pm 2}$ & $\nu_{6}+2 \nu_{10}$ & $\nu_{6}+2 \nu_{16}$ \\
\hline$\nu_{0}$ & 38606.089 & 39252.766 & 38560.794 & $\begin{array}{l}39771.392^{a)} \\
39772.590^{b)} \\
39771.990^{c}\end{array}$ & $\begin{array}{l}39080.831^{d)} \\
39084.115^{\text {e) }} \\
39082.368^{f)}\end{array}$ \\
\hline$B_{y}^{\prime}$ & 0.181778 & 0.181741 & 0.181660 & 0.181864 & 0.181745 \\
\hline$C_{\nu}^{\prime}$ & 0.090842 & 0.090949 & 0.091189 & 0.090968 & 0.091181 \\
\hline $10^{2} C \zeta_{6}$ & $5.25837(2)$ & - & - & $4.80256(10)$ & $5.08388(20)$ \\
\hline $10^{2} C \zeta_{t}$ & - & $0.10101(11)$ & $0.00273(63)$ & $0.26004(8)$ & $-0.03707(10)$ \\
\hline $10^{4} q_{6}$ & $0.1753(20)$ & - & - & 0.1753 * & $0.1753^{\circ)}$ \\
\hline $10^{4} q_{t}$ & - & $3.1404(150)$ & - & $2.7442(59)$ & $-2.0198(102)$ \\
\hline$S / R$ & - & - & - & $1.57512(1)$ & $0.46846(1)$ \\
\hline $10^{5} \lambda$ & - & - & - & $-7.099(23)$ & $-1.705(50)$ \\
\hline$\zeta_{6} \approx C \zeta_{6} / C_{v}^{\prime}$ & 0.5788 & - & - & 0.5279 & 0.5576 \\
\hline$\zeta_{t} \approx C \zeta_{t} / C_{\nu}^{\prime}$ & - & 0.0111 & 0.0003 & 0.0286 & -0.0041 \\
\hline$C_{\nu}^{\prime}-B_{\nu}^{\prime} / 2$ & -0.000047 & 0.000078 & 0.000359 & 0.000036 & 0.000309 \\
\hline$\Delta_{v}^{\prime}$ & 0.0960 & -0.1601 & -0.7313 & -0.0731 & -0.6276 \\
\hline $10^{3} \sigma$ & 0.37 & 0.55 & 0.61 & 0.54 & 0.68 \\
\hline$N$ & 353 & 164 & 81 & 392 & 285 \\
\hline
\end{tabular}

Spectroscopic constants of $S_{1}$ benzene determined in this work. The index $t$ can be either 10 or 16 , depending on the particular state. Values of $\zeta$ are dimensionless, the rotational defect $\Delta_{v}^{\prime}$ is given in amu $\AA^{2}$, and $N$ is the number of lines used in the fit. All other values are in $\mathrm{cm}^{-1} . \sigma$ is the standard deviation of the fit. Uncertainties (where given) are in units of the last digit

a) $\nu_{6}^{\mp}+2 \nu_{10}^{0}$. b) $\nu_{6}^{ \pm}+2 \nu_{10}^{ \pm 2}$. c) $\nu_{6}^{\mp}+2 \nu_{10}^{ \pm 2}$. d) $\nu_{6}^{\mp}+2 \nu_{16}^{0}$. ${ }^{\text {e) }} \nu_{6}^{ \pm}+2 \nu_{16}^{\mp 2}$. ${ }^{\text {f) }} \nu_{6}^{\mp}+2 \nu_{16}^{\mp 2}$. "s) Constrained value. 
change in the magnitude of the Coriolis term in the energy formula (1) which is known to dominate the rotational structure in vibronic bands of benzene [15]. For the $\nu_{6}^{\mp}$ state the value of $\zeta$ is quite sizeable, but for the out-of-plane mode $\nu_{10}$ it is expected to be close to zero. Keeping this fact in mind, assignment of the experimental spectrum was straightforward. Some of the assignments of the ${ }^{\mathbf{P}} \mathbf{P}$ branch lines are indicated above the experimental spectrum in fig. 1.

For the treatment of the upper states the appropriate matrix element for rotational l-type resonance [30],

$$
\begin{aligned}
& \left\langle l_{10}, J, K\left|h_{2}^{\dagger}\right| l_{10}-2, J, K-2\right\rangle \\
& =\sqrt{\frac{1}{2}} q_{10}^{+} F(J, K) F(J, K-1),
\end{aligned}
$$

was included. In addition, a fourth-order matrix element [30],

$$
\left\langle l_{10}, J, K+1\left|h_{4}^{\dagger}\right| l_{10}+4, J, K-1\right\rangle
$$

would be expected to couple the $2 \nu_{10}^{-2}(J, K+1)$ and $2 \nu_{10}^{+2}(J, K-1)$ states, but this could not be determined from the observed spectrum and its value was therefore constrained to zero.

With the matrix elements (3) and (4) the Hamiltonian matrix shown in table 3 was constructed and used for the least-squares refinement of the constants. The Coriolis terms in the diagonal elements were again determined according to the recipe of Hoy and Mills [29]. The constants resulting from the fit are included in table $2 . \zeta_{10}$ is indeed quite small as expected. A tentative value of $39253.04 \mathrm{~cm}^{-1}$ was obtained for $\nu_{0}\left(2 \nu_{10}^{0}\right)$. A calculated spectrum is shown in fig. 1 (lower trace) to demonstrate the excellent agreement between the observed and calculated line positions and intensities.

\subsubsection{The $16_{0}^{2}$ band}

Description of the transition to the $2 \nu_{16}$ state is quite similar to the situation for the $2 \nu_{10}$ state dis-

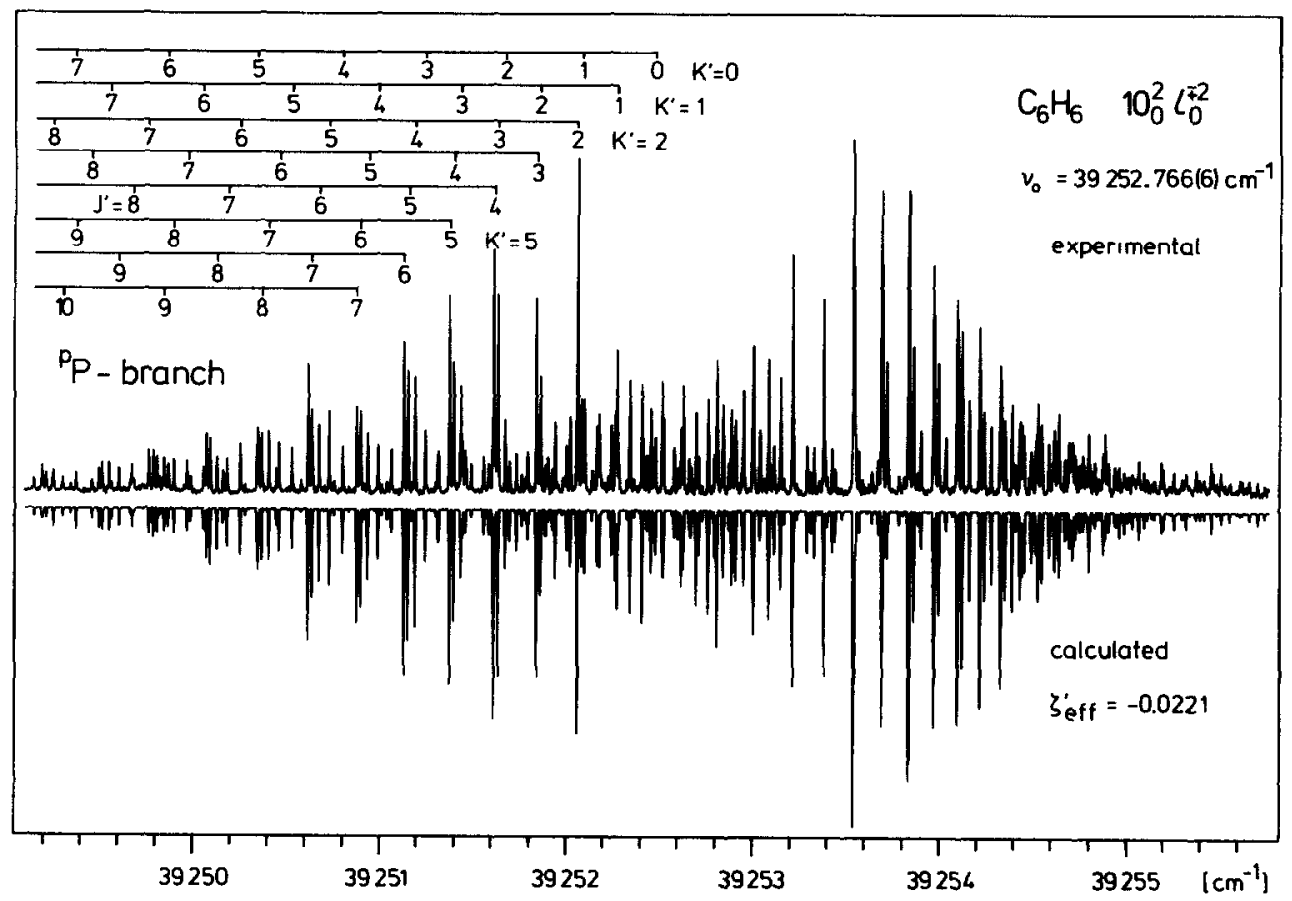

Fig. 1. Rotationally resolved electronic spectrum of $\mathrm{C}_{6} \mathrm{H}_{6}$. Upper trace: Fluorescence excitation spectrum of the $102 l_{0}^{2}$ band at a rotational temperature of $6.5 \mathrm{~K}$ and a linewidth of $0.0043 \mathrm{~cm}^{-1}$. Lower trace: Spectrum calculated with the constants obtained in the analysis of the experimental spectrum. The spectrum is displayed upside-down for easier comparison. Above the experimental spectrum some of the assignments of the ${ }^{P} \mathrm{P}$ branch are shown to illustrate the organization of the spectrum. 
Table 3

Hamiltonian matrix used for the treatment of the $10_{0}^{2} l_{0}^{\mp 2}$ band of $\mathrm{C}_{6} \mathrm{H}_{6}$. ${ }^{*}$ denotes a fourth-order coupling matrix element between the $2 \nu_{10}^{-2}$ and $2 \nu_{10}^{+2}$ states that could not be determined and was therefore constrained to zero

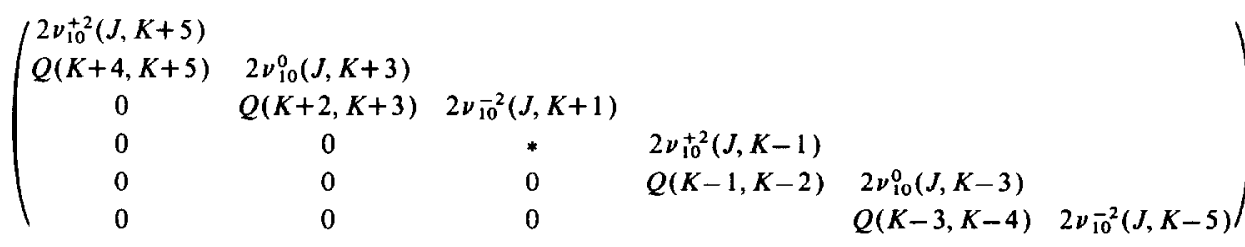

$2 \nu_{10}^{\mp 2}(J, K)=\nu_{0}\left(2 \nu_{10}^{\mp 2}\right)+\Delta B\left[\left(J(J+1)-K^{2}\right]+\Delta C K^{2} \mp 4 K\left(C \zeta_{10}\right)\right.$,

$2 \nu_{10}^{0}(J, K)=\nu_{0}\left(2 \nu_{10}^{0}\right)+\Delta B\left[J(J+1)-K^{2}\right]+\Delta C K^{2}$,

$Q(K, K+1)=(1 / \sqrt{2}) q_{10}^{+} F(J, K) F(J, K+1), \quad F(J, K)=[J(J+1)-K(K-1)]^{1 / 2}$

cussed above. The only difference is that the $(+l)$ state is now the $2 \nu_{16}^{+2}$ state and the $(-l)$ state is the $2 \nu_{16}^{-2}$ state, i.e. the observed band is correctly identified as the $16_{0}^{2} l_{0}^{ \pm 2}$ transition. This is due to the fact that the symmetry of $\nu_{10}$ is $\mathrm{E}_{1 \mathrm{~g}}$ while $\nu_{16}$ is of $\mathrm{E}_{2 \mathrm{u}} \mathrm{sym}$ metry. An experimental spectrum was available only for part of this band and therefore the effect of the rotational $l$ resonance was not included in the analysis. Instead, the energy of the $2 \nu_{16}^{ \pm 2}(J, K)$ states was described by

$$
\begin{aligned}
& E(J, K)=\nu_{0}+B_{\nu}^{\prime}\left[J(J+1)-K^{2}\right] \\
& \quad+C_{\nu}^{\prime} K^{2} \mp 2 K C_{\nu}^{\prime} \zeta_{\text {eff }}^{\prime} .
\end{aligned}
$$

The minus sign in eq. $(5)$ is to be used for the $(+l)$ substate and the plus sign for the $(-l)$ one. Furthermore it is found that $\zeta_{\text {eff }}^{\prime}=+2 \zeta_{16}$. The appearance of the $16_{0}^{2} l_{0}^{ \pm 2}$ band is very similar to that of the $10_{0}^{2} l_{0}^{\mp 2}$ band. This indicates right away that $\zeta_{16}$ is very small in magnitude, in accord with the out-of-plane character of $\nu_{16}$. Indeed, the result of the fit of the spectroscopic constants to the observed line positions (shown in table 2) gives $\zeta_{16}=0.0003$.

The fairly large rotational defect,

$\Delta_{v}^{\prime}=\frac{h}{8 \pi^{2} c}\left(\frac{1}{C_{v}^{\prime}}-\frac{2}{B_{v}^{\prime}}\right)$,

of $-0.7313 \mathrm{amu} \AA^{2}$ found for the $2 \nu_{16}$ state might at first sight be taken as indication of a perturbation in the $16_{0}^{2} l_{0}^{ \pm 2}$ band which was not properly accounted for. However, comparison with the recent calculation of rotational defects in $S_{0}$ benzene [31] shows that excitation of the $\nu_{16}$ mode should indeed produce a sizeable rotational defect.

\subsection{Transitions to combination states}

Both the $\nu_{6}+2 \nu_{10}$ and the $\nu_{6}+2 \nu_{16}$ states are sixfold degenerate and they give rise to two bands each in the electronic spectrum [17]. Since previous authors have attempted to analyze the rotational contours of these bands separately, but have encountered serious problems doing so [ 15,16$]$, we will first perform a similar analysis for the rotationally resolved spectra available now. This will help to understand why these earlier attempts could not be successful and give important hints on the correct Hamiltonian for the treatment of the combination states considered.

\subsubsection{Separate analysis of the $6_{0}^{1} 10_{0}^{2}$ subbands}

The symmetry of the vibrational angular momentum substates of the $\nu_{6}+2 \nu_{10}$ vibronic state is $\mathrm{e}_{1 \mathrm{u}}$ for the $\nu_{6}^{\mp}+2 \nu_{10}^{0}$ and $\nu_{6}^{ \pm}+2 \nu_{10}^{ \pm 2}$ components and $b_{1 u}+b_{2 u}$ for $\nu_{6}^{\mp}+2 \nu_{10}^{ \pm 2}$ (all including the $B_{2 u}$ symmetry of the $S_{1}$ electronic state). Therefore, the $6_{0}^{1} l_{0}^{\mp} 10_{0}^{2} l_{0}^{0}$ transition allowed by first-order Herzberg-Teller coupling and the third-order $6_{0}^{1} l_{0}^{ \pm} 10_{0}^{2} l_{0}^{ \pm 2}$ transition are expected to be seen as perpendicular bands, the latter being much weaker. From dispersed emission spectra at jet conditions it was found [17] that indeed two bands with vibrational assignment $6_{0}^{1} 10_{0}^{2}$ appear at $39762 \mathrm{~cm}^{-1}$ and $39780 \mathrm{~cm}^{-1}$. However, these two bands are of nearly equal intensity and the splitting of $18 \mathrm{~cm}^{-1}$ is unexpectedly large.

If the simple energy formula ( 5 ) is used for the description of each upper state, i.e. if the splitting of the two $e_{1 u}$ states is considered to be purely vibronic, a prediction can be made about the rotational struc- 
ture of these bands. We just have to calculate the expected value of $\zeta_{\text {eff }}^{\prime}$ from the known values of $\zeta_{6}$ and $\zeta_{10}$ (see table 2) and remember that the rotational structure of the vibronic bands of benzene is almost exclusively determined by this quantity [15]. For the $\nu_{6}^{\mp}+2 \nu_{16}^{0}$ state we expect $\zeta_{\text {eff }}^{\prime} \approx-\zeta_{6}=-0.5788$ and the band should be very similar to the $6_{0}^{1} l_{0}^{\mp}$ band. For the $\nu_{6}^{\frac{1}{6}}+2 \nu_{10}^{ \pm 2}$ state $\zeta_{\text {eff }}^{\prime} \approx \zeta_{6}+2 \zeta_{10}=+0.6010$ is expected and the rotational structure of the band should be quite different from both the $6_{0}^{1} l_{0}^{\mp}$ band reported previously [11] and the $10_{0}^{2} l_{0}^{\mp 2}$ band shown in fig. 1 .

We were now able to record the rotationally resolved fluorescence excitation spectrum of both bands at a rotational temperature of $6 \mathrm{~K}$. The spectra are shown in fig. 2. The lower-energy band is labeled as a-band, the higher energy one as b-band since the assignment of the vibrational angular momentum character is not yet known. Neither band is in agreement with the above explained expectation of the rota- tional structure. Instead, both are very similar to the $10_{0}^{2} l_{0}^{\mp 2}$ band in appearance and we can conclude that $\zeta_{\text {.eff }}^{\prime}$ has to be close to zero for both bands. The rotationally resolved spectra confirm the old impressions from Doppler-limited contour spectra [15,16].

To get a more quantitative understanding of the situation, the two $6_{0}^{1} 10_{0}^{2}$ bands were assigned and the effective spectroscopic constants determined according to eq. (5). The results of the separate fits for the a- and the b-band are shown in table 4 and a number of conclusions can be drawn from them:

(1) The standard deviation $\sigma$ of each fit is much larger than the one of the previously discussed bands. There $\sigma$ was about equal to the estimated accuracy of our frequency calibration. The large value of $\sigma$ does not stem from statistically distributed deviations, but rather a clear $J$ and $K$ dependence is found. We can hence conclude that the simple model used for the interpretation is not correct. However, it still is ac-

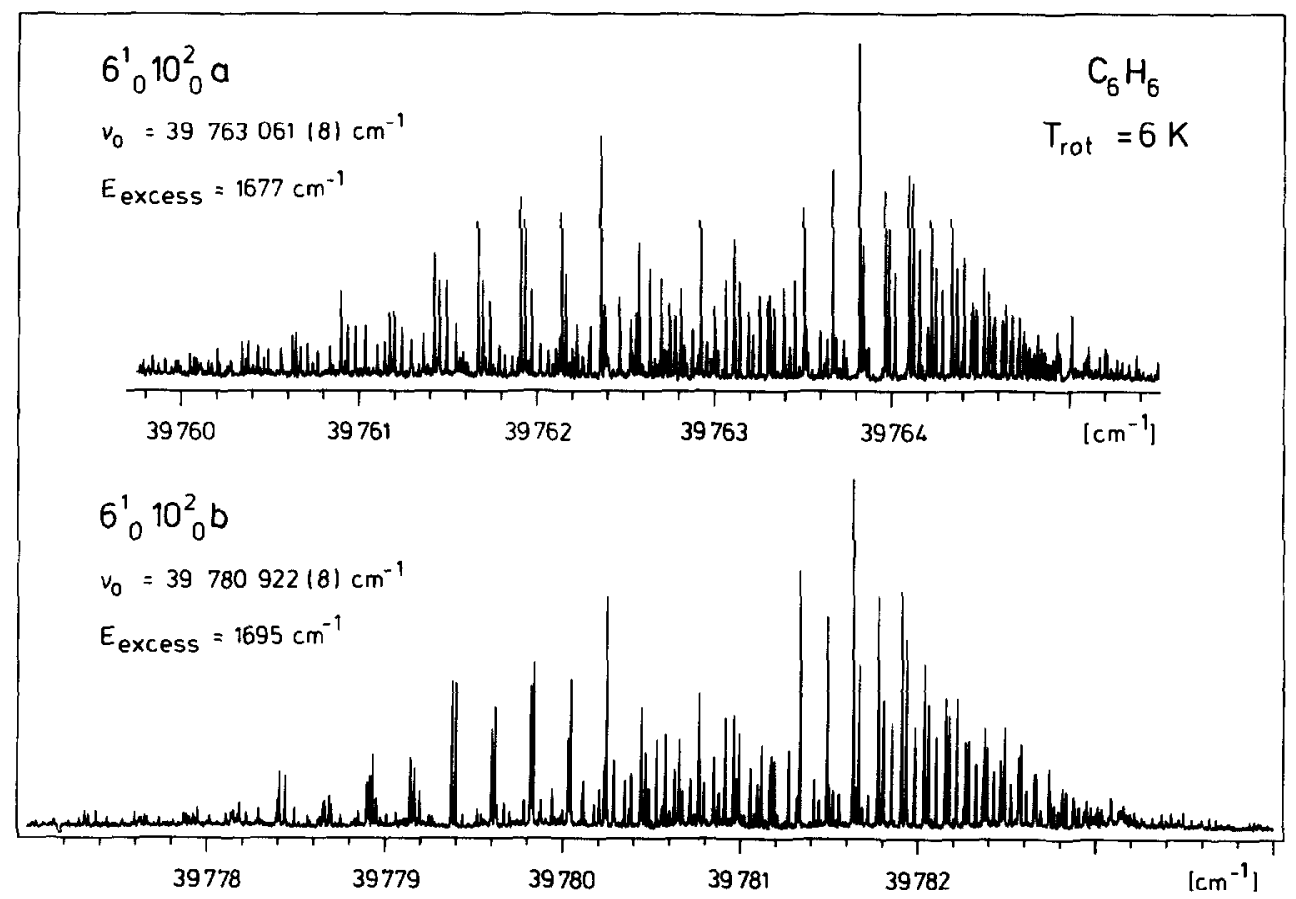

Fig. 2. Sub-Doppler fluorescence excitation spectrum of the two components a and $b$ of the $6_{0}^{1} 10_{0}^{2}$ vibronic transition of $\mathrm{C}_{6} \mathrm{H}_{6}$. Note the splitting of about $18 \mathrm{~cm}^{-1}$ between the two bands. The origin of this splitting and the identification of the two bands is discussed in the text. 
Table 4

Effective spectroscopic constants for the $6^{1} 10^{2}$ and $6^{1} 16^{2}$ states of $\mathrm{C}_{6} \mathrm{H}_{6}$ (in $\mathrm{cm}^{-1}$ ). Each state gives rise to two bands (labeled a and $\mathrm{b}$ ) in the electronic spectrum due to the splitting of the nominally degenerate vibrational angular momentum components. These bands are analyzed separately. For details see text. In addition to the value of the band origin $\nu_{0}$, the rotational constants $B_{\nu}^{\prime}$ and $C_{\nu}^{\prime}$, and the effective Coriolis coupling coefficient $\zeta_{\text {eff }}^{\prime}$, also the deviation $C_{v}^{\prime}-B_{v}^{\prime} / 2$ from the planarity condition and the resulting rotational defect $\Delta_{\nu}^{\prime}$ are given. $\sigma$ is the standard deviation of the fit and $N$ the number of lines used in the fit. $\Delta \nu$ denotes the observed splitting of the vibrational angular components

\begin{tabular}{lcccc}
\hline & $6^{1} 10^{2} \mathrm{a}$ & $6^{1} 10^{2} \mathrm{~b}$ & $6^{1} 16^{2} \mathrm{a}$ & $6^{1} 16^{2} \mathrm{~b}$ \\
\hline$\nu_{0}$ & 39763.061 & 39780.922 & 39079.356 & 39085.588 \\
$B_{v}^{\prime}$ & 0.181941 & 0.181778 & 0.181746 & 0.181722 \\
$C_{v}^{\prime}$ & 0.090398 & 0.091541 & 0.089941 & 0.092572 \\
$C_{v}^{\prime}-B_{v}^{\prime} / 2$ & -0.000572 & 0.000653 & -0.000932 & 0.001711 \\
$\Delta_{v}^{\prime}\left(\mathrm{amu} \AA^{2}\right)$ & 1.1735 & -1.3223 & 1.9227 & -3.4295 \\
$\zeta_{\text {eff }}^{\prime}$ & -0.0083 & 0.0654 & -0.2869 & 0.2913 \\
$10^{3} \sigma$ & 2.02 & 2.07 & 5.59 & 105 \\
$N$ & 209 & 198 & 180 & \\
$\Delta \nu\left(6^{1} 10^{2}\right)=17.861 \mathrm{~cm}^{-1}, \Delta \nu\left(6^{1} 16^{2}\right)=6.232 \mathrm{~cm}^{-1}$ & & & \\
\hline
\end{tabular}

curate enough to make the bands appear quite regular at Doppler-limited resolution.

(2) The values of $\zeta_{\text {eff }}^{\prime}$ are quite close to zero, contrary to expectation.

(3) The splitting of the band origins is 17.861 $\mathrm{cm}^{-1}$, in perfect agreement with the value deduced from the low-resolution experiments [17].

(4) The values of $B_{v}^{\prime}$, which should be equal according to theory, do differ slightly. The values of $C_{v}^{\prime}$, which should also be equal, differ quite strongly and large rotational defects $\Delta_{v}^{\prime}$ result for the two bands. Interestingly, one of them is positive and the other one negative.

All these results indicate that the simple modeling of the $\nu_{6}+2 \nu_{10}$ state performed so far is not sufficient. The expected large values and opposite signs of $\xi_{\text {eff }}^{\prime}$ seem to cancel and large effective rotational defects of opposite sign result. This situation bears a lot of resemblance to the Fermi dyad $\nu_{8} / \nu_{6}+\nu_{1}$ in ground state benzene $[5,6]$. However, a simple anharmonic resonance is not possible between the $\nu_{6}^{\mp}+2 \nu_{10}^{0}$ and $\nu_{6}^{ \pm}+2 \nu_{10}^{ \pm 2}$ states.

\subsubsection{Separate analysis of the $6_{0}^{1} 16_{0}^{2}$ subbands}

The situation of the possible $6_{0}^{1} 16_{0}^{2}$ transitions is quite similar to that discussed for the $6_{0}^{1} 10_{0}^{2}$ bands. Only changes in the signs of the $l$ quantum numbers result due to the differing symmetry of $\nu_{10}$ and $\nu_{16}$. The strong $6_{0}^{1} l_{0}^{\mp} 16_{0}^{2} l_{0}^{D}$ band should be characterized by $\zeta_{\text {eff }}^{\prime} \approx-\zeta_{6}=-0.5788$ and the weaker $6_{0}^{1} l_{0}^{ \pm} 16_{0}^{2} l_{0}^{\mp} 2$ band by $\zeta_{\text {eff }}^{\prime} \approx \zeta_{6}-2 \zeta_{16}=+0.5782$.

The two bands at $39078 \mathrm{~cm}^{-1}$ and $39084 \mathrm{~cm}^{-1}$ previously assigned as $6_{0}^{1} 16_{0}^{2}$ transitions [17] were measured with sub-Doppler resolution. The rotationally resolved two-photon ionization spectra are shown in fig. 3. The b-band is displayed at some magnification since it is considerably weaker [17]. Again, the expectations on the rotational structure given by the calculated values of $\zeta_{\text {eff }}^{\prime}$ cannot be confirmed. The aband appears somewhat intermediate between the $6_{0}^{1} l_{0}^{\mp}$ and the $10_{0}^{2} l_{0}^{\mp 2}$ bands.

This impression is confirmed by a detailed assignment and a fit according to eq. (5). The results are included in table 4 . The standard deviations of the fits are even worse than in the $6_{0}^{1} 10_{0}^{2}$ bands and the resulting rotational defects of opposite sign extremely large. Such large and differing values can certainly not be real for the different vibrational angular momentum components of the same vibronic state. The values of $\zeta_{\text {eff }}^{\prime}$ are about half of what is expected. In summary, the simple modeling of the bands again does not account properly for the observed rotational structure of the $6_{0}^{1} 16_{0}^{2}$ bands.

\subsubsection{Comparehensive analysis of the $6_{0}^{1} 10_{0}^{2}$ bands}

The separate analysis of the $6_{0}^{1} 10_{0}^{2}$ bands discussed above led to the conclusion that strong coupling between the substates of $\nu_{6}+2 \nu_{10}$ might be responsible 


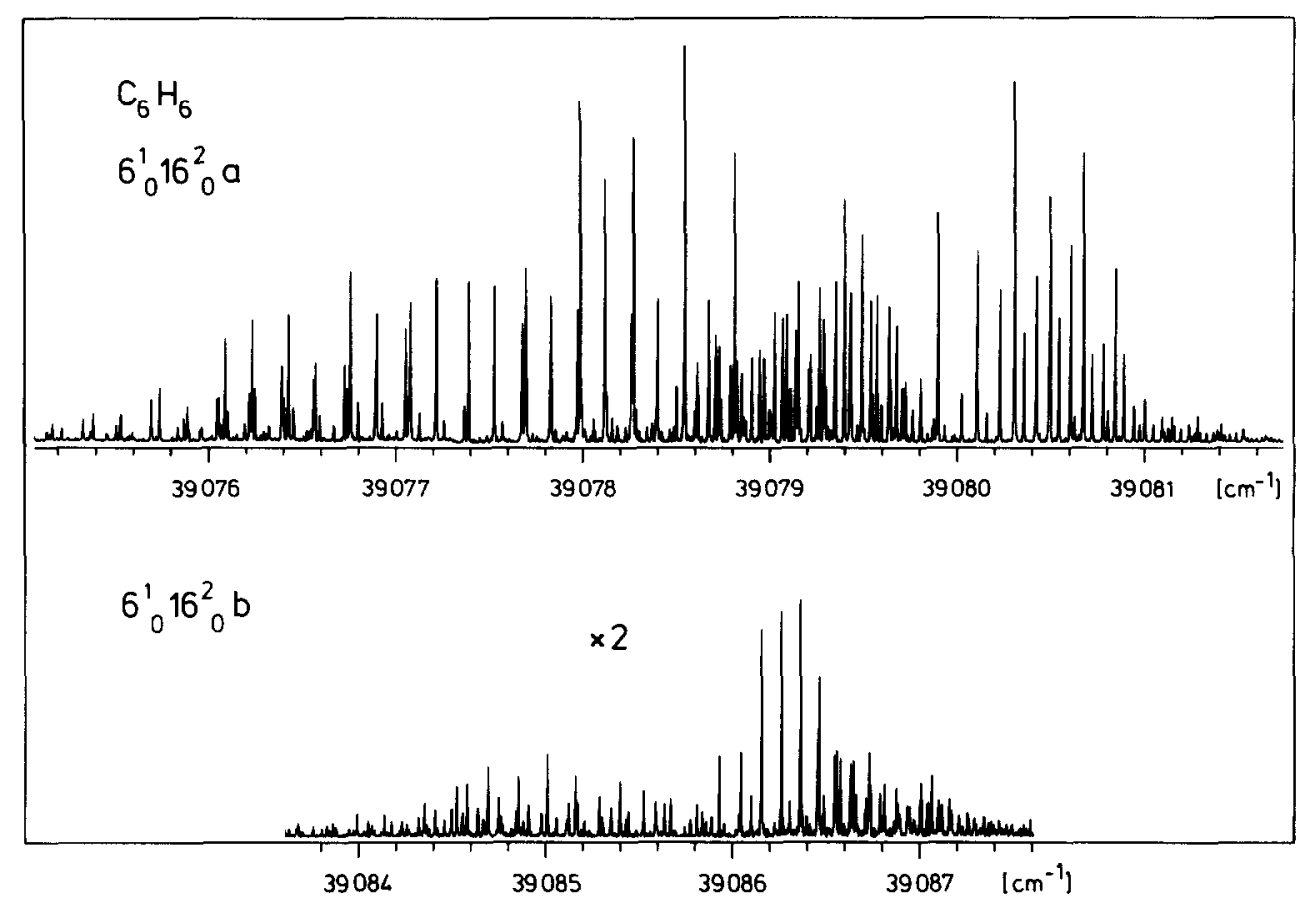

Fig. 3. Sub-Doppler two-photon ionization spectrum of the two components a and $b$ of the $6_{0}^{1} 16_{0}^{2}$ vibronic transition of $\mathrm{C}_{6} \mathrm{H}_{6}$. Note the splitting of about $6 \mathrm{~cm}^{-1}$ between the two bands. For clarity the weaker b-band is displayed with increased vertical scale.

for the observed situation. Selective couplings of the substates to other $S_{1}$ states, as was postulated previously [19] seems unlikely due to the very symmetric appearance of the perturbations. The rotational l-type resonances, eqs. (2) and (3), are certainly too weak to account for the large splitting observed and anharmonic resonance is not possible for states differing in $l$ quantum numbers. What is left to third order of approximation are therefore vibrational l-type resonances [30]. For the states under discussion it can be determined with the help of the Amat rule [30] that the matrix element

$$
\begin{aligned}
& \left\langle l_{6}, l_{10}\left|h_{2}^{+}\right| l_{6} \pm 2, l_{10} \pm 2\right\rangle \\
& =S_{6,10}\left[\left(v_{6}+l_{6}+1 \pm 1\right) \times\left(v_{6}-l_{6}+1 \mp 1\right)\right. \\
& \left.\quad \times\left(v_{10}+l_{10}+1 \pm 1\right)\left(v_{10}-l_{10}+1 \mp 1\right)\right]^{1 / 2}
\end{aligned}
$$

should be nonvanishing. The vibrational factor in eq. (7) amounts to $\sqrt{32}$ and we can hence expect even a reasonably sized $S_{6,10}$ to cause a large effect. We therefore included the matrix element

$$
\begin{aligned}
& \left\langle l_{6}, l_{10}\left|h_{2}^{\dagger}\right| l_{6} \pm 2, l_{10} \pm 2\right\rangle \\
& =\sqrt{32} S_{6,10}+\lambda_{6,10}\left[J(J+1)-K^{2}\right]
\end{aligned}
$$

and the rotational $l$-type resonances (2) and (3) in the description of the $\nu_{6}+2 \nu_{10}$ states. The $J$-dependent correction term $\lambda_{6,10}$ is the sum of two fourthorder terms [30] which cannot be determined separately. The resulting Hamiltonian matrix is shown in table 5 . It should be noted that the same values of $B_{v}^{\prime}$ and $C_{v}^{\prime}$ are assumed for all substates.

Using this Hamiltonian, a combined fit of the parameters to all the observed lines in the $a$ - and b-band of the $6_{0}^{1} 10_{0}^{2}$ transition was possible. The resulting values of the spectroscopic constants are included in table 2 . In addition, the following points are noted:

(1) The standard deviation of the fit is comparable to our accuracy of calibration. There are no systematic deviations as in the separate fits and no strong correlation between any of the parameters is found. 
Table 5

Hamiltonian matrix used for the treatment of the $6_{0}^{1} 10_{0}^{2}$ bands of $\mathrm{C}_{6} \mathrm{H}_{6}$

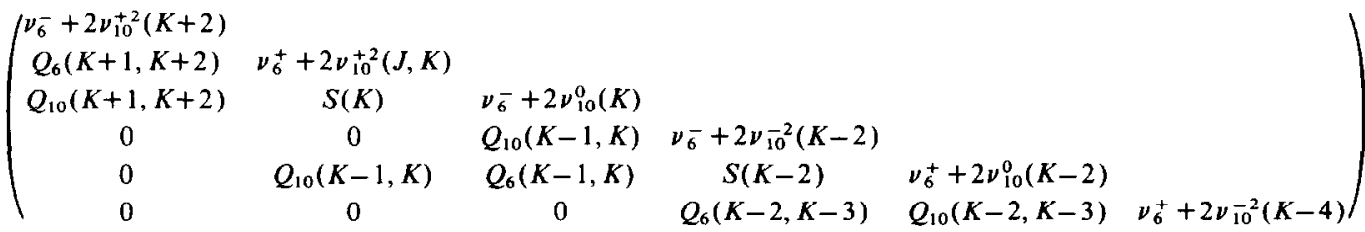

$$
\begin{aligned}
& \nu_{6}^{\mp}+2 \nu_{10}^{0}(K)=\nu_{0}\left(\nu_{6}^{\mp}+2 \nu_{10}^{0}\right)+\Delta B\left[J(J+1)-K^{2}\right]+\Delta C K^{2} \pm 2 K\left(C \zeta_{6}\right) \text {, } \\
& \nu_{6}^{ \pm}+2 \nu_{10}^{ \pm 2}(K)=\nu_{0}\left(\nu_{6}^{ \pm}+2 \nu_{10}^{ \pm 2}\right)+\Delta B\left[J(J+1)-K^{2}\right]+\Delta C K^{2} \mp 2 K\left(C \zeta_{6}\right) \mp 4 K\left(C \zeta_{10}\right) \text {, } \\
& \nu_{6}^{\mp}+2 \nu_{10}^{ \pm 2}(K)=\nu_{0}\left(\nu_{6}^{\mp}+2 \nu_{10}^{ \pm 2}\right)+\Delta B\left[J(J+1)-K^{2}\right]+\Delta C K^{2} \mp 2 K\left(C \zeta_{6}\right) \pm 4 K\left(C \zeta_{10}\right) \text {, } \\
& Q_{6}(K, K+1)=\frac{1}{2} q_{6}^{-} F(J, K) F(J, K+1), \quad Q_{10}(K, K+1)=(1 / \sqrt{2}) q_{10}^{+} F(J, K) F(. I, K+1) \text {. } \\
& F(J, K)=[J(J+1)-K(K-1)]^{1 / 2}, \quad S(K)=\sqrt{32} S_{6,10}+\lambda_{6,10}\left[J(J+1)-K^{2}\right]
\end{aligned}
$$

(2) The resulting value of the rotational defect of the $\nu_{6}+2 \nu_{10}$ state is quite small. It is very close to the sum of the rotational defects of the $\nu_{6}$ and $2 \nu_{10}$ states as would be expected for a small rotational defect of the vibrationless $S_{1}$ state (see below).

(3) The values of $\zeta_{6}$ and $\zeta_{10}$ found from the analysis of this complex level system are rather close to those obtained from the $6_{0}^{1}$ and $10_{0}^{2}$ bands.

(3) $q_{6}^{-}$was constrained to the value obtained from the analysis of the $6{ }_{0}^{1} l_{0}^{\mp}$ band. The value of $q_{10}^{+}$turns out quite close to the one found in the $10_{0}^{2} l_{0}^{\mp 2}$ band.

(4) The separation of the unperturbed $\nu_{6}^{\mp}+2 \nu_{10}^{0}$ and $\nu_{6}^{ \pm}+2 \nu_{10}^{ \pm 2}$ substates is reduced to $1.198 \mathrm{~cm}^{-1}$. This is a much more reasonable anharmonic splitting than the large effective splitting postulated before [17]. The effective separation of the bands is mostly due to the vibrational $l$-type resonance.

In summary, we believe all these observations to be overwhelming proof for the correctness of our Hamiltonian. It is therewith shown that vibrational l-type resonance can effectively couple different vibrational angular momentum components of combination states of benzene. The magnitude of $S_{6,10}$ itself is not extremely large, but the prefactor of $\sqrt{32}$ makes the resulting effect quite sizeable.

\subsubsection{Comprehensive analysis of the $6_{0}^{1} 16_{0}^{2}$ bands}

Transfer of the comprehensive analysis of the $6_{0}^{1} 10_{0}^{2}$ bands to the $6_{0}^{1} 16_{0}^{2}$ bands is straightforward. Because of the symmetry of $\nu_{16}$ the appropriate matrix element for vibrational $l$-type resonance is [30]

$$
\begin{aligned}
& \left\langle l_{6}, l_{16}\left|h_{2}^{\dagger}\right| l_{6} \pm 2, l_{16} \mp 2\right\rangle \\
& \quad=R_{6,16}\left[\left(v_{6}+l_{6}+1 \pm 1\right)\left(v_{6}-l_{6}+1 \mp 1\right)\right. \\
& \left.\quad \times\left(v_{16}+l_{16}+1 \mp 1\right)\left(v_{16}-l_{16}+1 \pm 1\right)\right]^{1 / 2}
\end{aligned}
$$

and this results in the matrix element

$$
\begin{aligned}
& \left\langle l_{6}, l_{16}\left|h_{2}^{\dagger}\right| l_{6} \pm 2, l_{16} \mp 2\right\rangle \\
& =\sqrt{32} R_{6,16}+\lambda_{6,16}\left[J(J+1)-K^{2}\right]
\end{aligned}
$$

to be included in the Hamiltonian. In addition, the rotational $l$-type resonance ( 3 ) has be replaced by

$$
\begin{aligned}
& \left\langle l_{16}, J, K\left|h_{2}^{\dagger}\right| l_{16}+2, J, K-2\right\rangle \\
& =\sqrt{\frac{1}{2}} q_{16}^{-} F(J, K) F(J, K-1) .
\end{aligned}
$$

The resulting Hamiltonian matrix is shown in table 6. Again note that the rotational constants are set equal for all the substates.

The combined fit of the parameters to all the lines of the a- and b-band of the $6_{0}^{1} 16_{0}^{2}$ transition resulted in the values shown in table 2 . The fit is again good despite the increased difficulties encountered with these bands in the separate analysis. The values of $\zeta_{6}$ and $\zeta_{16}$ are remarkably close to those obtained from the component bands. The rotational defect is sizeable, but quite similar to that of the $2 \nu_{16}$ state. The value of $q_{16}^{-}$seems reasonable. All these points can be taken as proof for the correct interpretation of the complicated level scheme.

The value of $R_{6,16}$ is smaller than that of $S_{6,10}$ by a factor of 3.4. On the other hand, the deperturbed splitting of $3.284 \mathrm{~cm}^{-1}$ between the $\nu_{6}^{\mp}+2 \nu_{16}^{0}$ and $\nu_{6}^{ \pm}+2 \nu_{16}^{\mp 2}$ substates is larger by a factor of 2.7. This 
Table 6

Hamiltonian matrix used for the treatment of the $6_{0}^{1} 16_{0}^{2}$ bands of $\mathrm{C}_{6} \mathrm{H}_{6}$

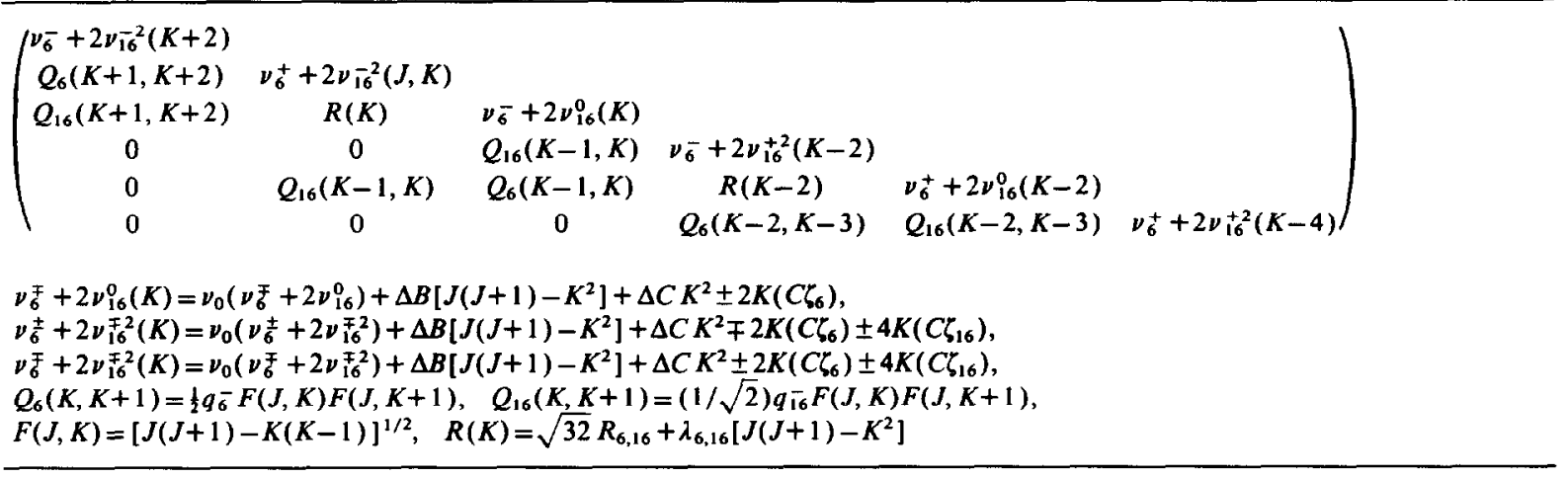

results in less mixing of the zero-order wave functions and a smaller relative intensity of the b-band than in the $6_{0}^{1} 10_{0}^{2}$ transition. To illustrate this point, we have calculated the spectrum of the $6_{0}^{1} 16_{0}^{2}$ transition with the correct Hamiltonian and the newly determined spectroscopic constants under the assumption that only the $\nu_{6}^{\mp}+2 \nu_{16}^{0}$ state contributes any oscillator strength. The result is shown in the upper trace of fig. 4 and compared to the experimental spectrum (lower trace). It is clearly seen that the bband (at higher energy) is much weaker. In this band we also find that the $P$ branch is considerably weaker than the $\mathbf{R}$ branch. This is due to the fact that the $(+l)$ states which contribute most of the intensity of the $\mathrm{R}$ branch are more heavily mixed. The mixing between the states will be discussed in more detail below.

\section{Discussion}

The comprehensive analysis of the $6_{0}^{1} 10_{0}^{2}$ and $6_{0}^{1} 16_{0}^{2}$ bands has provided us with a precise picture of the zero-order states and the couplings between them. The matrix formalism used for this analysis complicates the physical understanding of the level structure and we will therefore proceed now to give a qualitative description of the level shifts encountered. This should help to understand the observed band structure and the level mixing.

Inspection of the Hamiltonians (tables 5 and 6) and the newly determined values of the spectroscopic constants (table 2) tells us that coupling to the $\nu_{6}^{\mp}+2 \nu_{10}^{ \pm 2}$ and $\nu_{6}^{\mp}+2 \nu_{16}^{\mp 2}$ substates is quite small compared to the coupling between the other two substates of each combination state. We can therefore neglect these states in the present qualitative discussion. We can also neglect the rotational $l$-type resonances (2), (3) and (11) since they are much weaker than the vibrational ones. What is left, is strong coupling by the vibrational l-type resonance $S_{6,10}$ between the $\nu_{6}^{-}+2 \nu_{10}^{0}$ and $\nu_{6}^{+}+2 \nu_{10}^{+2}$ states and between the $\nu_{6}^{+}+2 \nu_{10}^{0}$ and $\nu_{6}^{-}+2 \nu_{10}^{-2}$ states and correspondingly for the $\nu_{6}+2 \nu_{16}$ states.

The spacing between the $\nu_{6}^{-}+2 \nu_{10}^{0}(J, K)$ and $\nu_{6}^{+}+2 \nu_{10}^{+2}(J, K)$ zero-order rovibronic states is independent of $J$ but depends strongly on $K$-due to the opposite sign of the Coriolis term $2 K\left(C \zeta_{6}\right)$. The term $4 K\left(C \zeta_{10}\right)$ can be neglected at present. The spacing is $1.198 \mathrm{~cm}^{-1}$ for $K=0$ and decreases by $0.1921 \mathrm{~cm}^{-1}$ for an increase in $K$ by 1 . This results in a crossing of the term curves between $K=5$ and $K=6$. The vibrational $l$-type resonance causes repulsion of the levels that is strongest for the closest spaced levels, i.e. it is $K$ dependent. This $K$ dependence is seen as a reduction of the term linear in $K$, the Coriolis term, in the separate analysis of the subbands. However, the correction is not purely linear in $K$ and a large $K^{2}$ term is left which is interpreted as a change in $C_{v}^{\prime}$ and a smaller one of opposite sign in $B_{v}^{\prime}$. The observed large rotational defect results. Similarly, the spacing between the $\nu_{6}^{+}+2 \nu_{10}^{0}(J, K)$ and $\nu_{6}^{-}+2 \nu_{10}^{-2}(J, K)$ states increases by $0.1921 \mathrm{~cm}^{-1}$ for an increase in $K$ by 1 and less repulsion results for higher $K$ values. The influence on the effective constants is as before. 


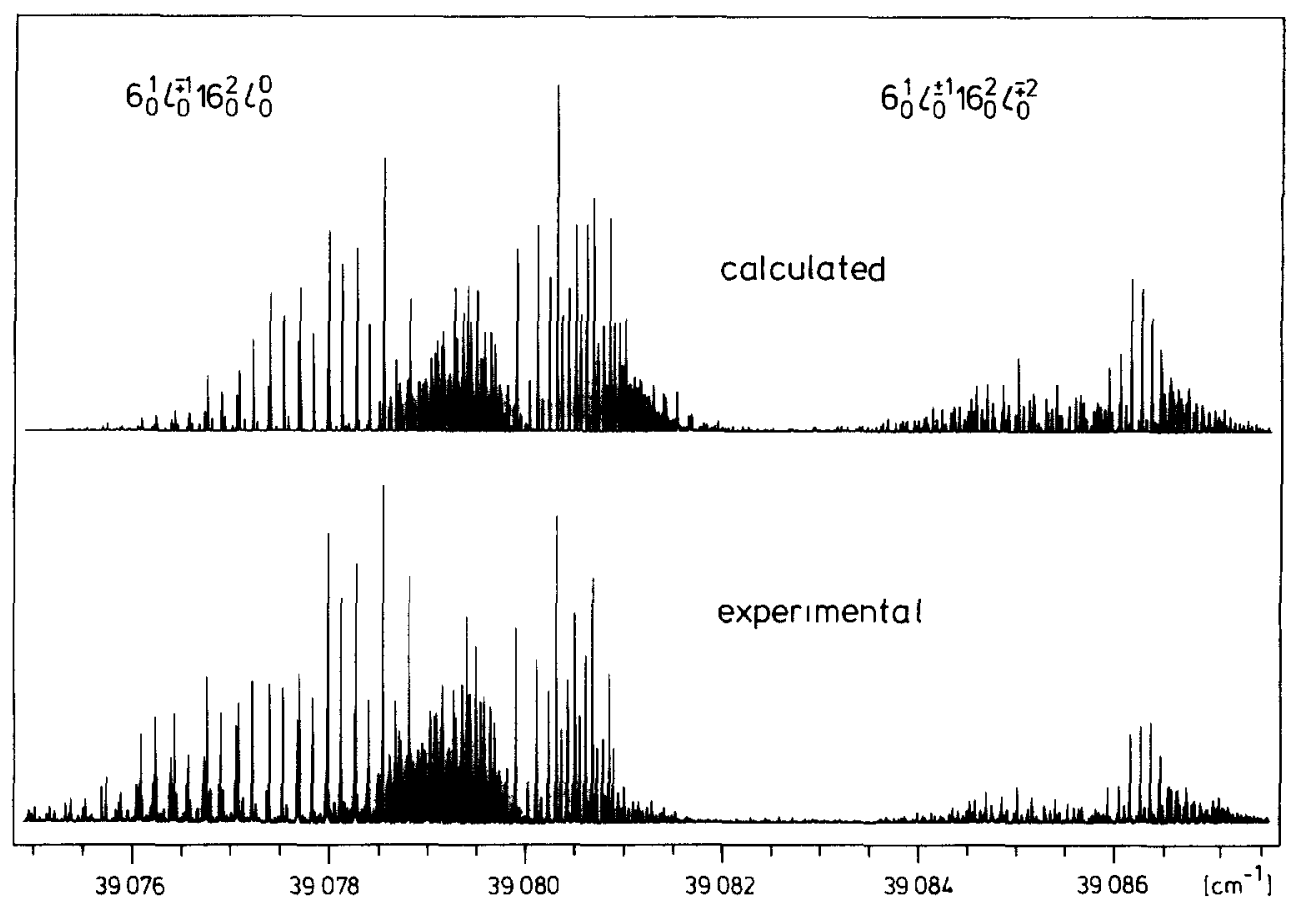

Fig. 4. Comparison between the experimental spectrum of the $6_{0}^{1} 16_{0}^{2}$ vibronic transition and the spectrum calculated according to the detailed analysis explained in the text. The appearance of the two subbands leading to differing vibrational angular momentum components of the $6^{1} 16^{2}$ state is due to strong vibrational $l$-type resonance and the consequent mixing of the zero-order states.

For the $\nu_{6}+2 \nu_{16}$ states the situation is similar. The $\nu_{6}^{+}+2 \nu_{16}^{0}(J, K)$ and $\nu_{6}^{-}+2 \nu_{16}^{+2}(J, K)$ states move apart with increasing value of $K$. The $\nu_{6}^{-}+$ $2 \nu_{16}^{0}(J, K)$ and $\nu_{6}^{+}+2 \nu_{16}^{-2}$ states move closer together as $K$ increases, but due to the larger zero-order splitting the crossing does not occur until about $K=17$, a value not observed under jet conditions. The coupling element $R_{6,16}$ is also smaller than $S_{6,10}$ and overall smaller but still important shifts of states and changes between the zero-order and the effective constants result.

In summary, in both the $6_{0}^{1} 10_{0}^{2}$ and the $6_{0}^{1} 16_{0}^{2}$ transitions we see the effects of strong level crossings. The rotationally independent matrix elements (7) and (9) have a strong influence on the observed rotational structure due to the opposite signs of the Coriolis terms for the zero-order states.

So far we have only discussed the spectral shifts caused by the couplings. However, the couplings also cause strong mixing of the zero-order wavefunctions. To discuss the mixing we want to define the content of a zero-order function in the eigenfunction as the square of the appropriate expansion coefficient.

The content of the $\nu_{6}^{-}+2 \nu_{10}^{0}$ state in the $(+l)$ states of the $6_{0}^{1} 10_{0}^{2} \mathrm{a}$ band is found to decrease from 0.528 for $K=1$ to 0.499 for $K=6$ and further beyond, with the balance due to the $\nu_{6}^{+}+2 \nu_{10}^{+2}$ state. In the bband the situation is just turned around. For the $(-l)$ states of the $6_{0}^{1} 10_{0}^{2} \mathrm{a}$ band we find the content of the $\nu_{6}^{+}+2 \nu_{10}^{0}$ state to increase from 0.521 for $K=0$ to 0.578 for $K=8$ and further beyond. The balance is due to the $\nu_{\sigma}^{-}+2 \nu_{10}^{-2}$ state and the situation again turned around for the $b$-band. There is no appreciable $J$ dependence of the mixing.

Due to the larger zero-order splitting and the weaker vibrational $l$-type resonance, mixing is not quite as strong in the upper states of the $6_{0}^{1} 16_{0}^{2}$ bands. The content of $\nu_{6}^{-}+2 \nu_{16}^{0}$ states in the $(+l)$ levels of the $6_{0}^{1} 16_{0}^{2} \mathrm{a}$ band is found to decrease from 0.750 for $K=1$ to 0.614 for $K=8$. The balance is due to the $\nu_{6}^{+}+2 \nu_{16}^{-2}$ state. At $K=17$ the mixing would be even. For the $(-l)$ states the content of the $\nu_{6}^{+}+2 \nu_{16}^{0}$ state 
increases from 0.761 for $K=0$ to 0.840 for $K=8$. The remainder is due to the $\nu_{6}^{-}+2 \nu_{16}^{+2}$ state. Again the situation is just turned around for the b-band since the upper states of the b-band are the companion eigenstates of the upper states of the a-band.

In summary, the mixing of the zero-order states $\nu_{6}^{\mp}+2 \nu_{10}^{0}$ and $\nu_{6}^{ \pm}+2 \nu_{10}^{ \pm 2}$ is nearly complete for the upper states of the observed $6_{0}^{1} 10_{0}^{2}$ transitions. The vibrational angular momentum character of the $S_{1}$ states accessed through these bands is no more defined. For the $\nu_{6}^{\mp}+2 \nu_{16}^{0}$ and $\nu_{6}^{ \pm}+2 \nu_{16}^{\mp 2}$ states mixing is about 3 to $l$ and it is still meaningful to assign $l$ quantum numbers to the observed $6_{0}^{1} 16_{0}^{2}$ bands. The lower energy a-band is indeed mainly the $6_{0}^{1} l_{0}^{\mp} 16_{0}^{2} l_{0}^{0}$ transition and the higher-energy $b$-band the $6_{0}^{1} l_{0}^{ \pm} 16_{0}^{2} l_{0}^{\mp 2}$ transition. These labels are also shown in fig. 4 .

As a last point, we want to discuss the possibility to extract some further spectroscopic constants of $S_{1}$ benzene from our precise results. We could hope to determine some of the anharmonic constants $x_{i j}$ and $g_{i j}$ for the vibrational modes investigated, but close inspection shows us that this is not possible due to the insufficient accuracy of the necessary reference values, particularly the pure electronic transition frequency. We can, however, determine values for $B_{0}^{\prime}$ and $C_{0}^{\prime}$ which are not available so far.

We start with the well known formula

$B_{v}^{\beta}=B_{e}^{\beta}-\sum_{s} \alpha_{s}^{\beta}\left(v_{s}+\frac{1}{2} d_{s}\right)=B \beta-\sum_{s} \alpha_{s}^{\beta} v_{s}$

where $B^{\beta}$ can be either $B$ or $C$. From (12) we can deduce that

$B_{6}^{\beta}=B^{\beta}\left(\nu_{6}\right)+B^{\beta}\left(2 \nu_{10}\right)-B^{\beta}\left(\nu_{6}+2 \nu_{10}\right)$.

This yields

$B_{0}^{\prime}=0.181655 \mathrm{~cm}^{-1}$,

$C_{0}^{\prime}=0.090823 \mathrm{~cm}^{-1}$

and a value of $\Delta_{0}^{\prime}=0.0092 \mathrm{amu} \AA^{2}$ for the rotational defect of the zero point $S_{1}$ state. An analogous cvaluation using the rotational constants of the states containing $2 \nu_{16}$ yields

$$
\begin{aligned}
& B_{0}^{\prime}=0.181693 \mathrm{~cm}^{-1}, \\
& C_{0}^{\prime}=0.090850 \mathrm{~cm}^{-1}
\end{aligned}
$$

and a value of $\Delta_{0}^{\prime}=-0.0071$ amu $\AA^{2}$ for the rotational defect.

We do not know how accurate eq. (12) is for the $S_{1}$ state of a large molecule like benzene. However, the differences in the rotational constants of various vibronic states are known [11] to be larger by more than one order of magnitude than the differences between (14) and (15). This may be an indication that the above determined values are fairly reliable. Until better estimates are available, they might be of some use. The fact that the rotational defect $\Delta_{0}^{\prime}$ turns out to be very small is certainly significant and a strong indication for the planarity of $S_{1}$ benzene.

\section{Summary and conclusion}

We have presented rotationally resolved sub-Doppler spectra of the $6_{0}^{1} 10_{0}^{2}$ and $6_{0}^{1} 16_{0}^{2}$ transitions of benzene. Both vibronic transitions give rise to two well separated bands which were previously believed to be due to transitions to different vibrational angular momentum substates of the sixfold degenerate combination states split by anharmonic terms. Detailed analysis of these bands shows that the $\nu_{6}^{\mp}+2 \nu_{10}^{0}$ and $\nu_{6}^{ \pm}+2 \nu_{10}^{ \pm 2}$ substates and the $\nu_{6}^{\mp}+2 \nu_{16}^{0}$ and $\nu_{6}^{ \pm}+2 \nu_{16}^{ \pm 2}$ substates are instead strongly coupled by vibrational $l$-type resonance. The rotationally independent resonances strongly influence the rotational structure of the transitions due to the differing contribution of Coriolis coupling to the energy of each zero-order state and are responsible for the observed overall splittings. Furthermore, the resonances are found to mix the vibrational angular momentum character of the substates nearly completely for the $\nu_{6}+2 \nu_{10}$ states and quite substantially for the $\nu_{6}+2 \nu_{16}$ states.

Since there is nothing special about the particular combination states investigated in this work, we believe that similarly strong resonances should also be present for many other multiply degenerate combination states in benzene. Contrary to the impression one gets from an inspection of the IR or UV spectrum, where transitions to combination states are not dominant, the multiply degenerate combination states present the majority of vibrational levels in a molecule like benzene already at moderate vibrational excess energies. The newly observed vibrational $l$-type 
resonances might therefore be just as important for the IVR and state mixing in the molecule as the well known anharmonic resonances and Coriolis coupling.

The implications for the decay behavior of electronically excited benzene could also be considerable. It was recently found that pumping the lower-energy band of the $6_{0}^{1} 16_{0}^{2}$ transition with limited spectral resolution results in an observed decay time of $73 \mathrm{~ns}$, while pumping the upper component gives a decay time of $80 \mathrm{~ns}$ [18]. Similarly, differing values of 44 ns and $55 \mathrm{~ns}$ were found for the $6_{0}^{1} 10_{0}^{2}$ transitions and for a number of bands in $\mathrm{C}_{6} \mathrm{D}_{6}$ [18]. Since the mixing of the vibrational angular momentum components was not known, this observation was interpreted as selective coupling of the two zero-order substates to other $S_{1}$ states [19]. The faster decay of the lower energy components was rationalized by the idea that it is more strongly mixed with states of higher excess energy possessing faster nonradiative decay rates [19].

In view of our new understanding we cannot confirm this concept. The nearly complete mixing of the substates is incompatible with the observed $20 \%$ differences, cven though we predict rotationally resolved decay time measurements to produce still larger variations. Such experiments have been successfully performed for other vibronic states of benzene [11-13]. The $\nu_{6}+2 \nu_{10}$ and $\nu_{6}+2 \nu_{16}$ states would indeed provide an unique opportunity for detailed investigation of the influence of mode mixing on the nonradiative decay of benzene. Variation of the degree of mixing could be obtained by variation of the $K$ quantum number and for fixed $K$ a variation in $J$ would cause a detuning with respect to the background states. Furthermore, both eigenstates resulting from the coupling of the zero-order states could be investigated by excitation through either one of the subbands. No other such perfect situation of smoothly varying level crossing has been found up to date in the spectrum of benzene and our theoretical understanding of the nonradiative decay of benzene would greatly profit from such an investigation. Particularly the clear observation of interference effects in the decay, which were both predicted theoretically [32] and invoked for the interpretation of highly perturbed spectra $[13,14]$, would be of great interest.

\section{Acknowledgements}

The authors are indebted to Professor Dr. Dr.h.c. E.W. Schlag and Professor Dr. H.J. Neusser for their continuous interest in this work. They want to thank Th. Knittel and Th. Weber for valuable experimental assistance and preliminary assignment of some of the spectra. Financial support from the Deutsche Forschungsgemeinschaft is gratefully acknowledged. One of us (ER) wants to thank NATO for support of travcl expenses through research grant No. 1018/83.

\section{References}

[1] J. Pliva and A.S. Pine, J. Mol. Spectry. 93 (1982) 209.

[2] J. Pliva and J.W.C. Johns, Canad. J. Phys. 61 (1983) 269.

[3] J. Pliva and J.W.C. Johns, J. Mol. Spectry. 107 (1984) 318.

[4] J. Pliva and A.S. Pine, J. Mol. Spectry. 126 (1987) 82.

[5] P. Esherick, A. Owyoung and J. Pliva, J. Chem. Phys. 83 (1985) 3311.

[6] J. Pliva, P. Esherick and A. Owyoung, J. Mol. Spectry. 125 (1987) 393.

[7] E. Riedle, H.J. Neusser and E.W. Schlag, J. Chem. Phys. 75 (1981) 4231

[8] E. Riedle, H. Stepp and H.J. Neusser, Chem. Phys. Letters 110 (1984) 452.

[9] H. Sicber, E. Riedle and H.J. Neusser, J. Chem. Phys. 89 (1988) 4620

[10] U. Schubert, E. Riedle and H.J. Neusser, J. Chem. Phys. 90 (1989) 5994.

[ 11 ] E. Riedle, Th. Knittel, Th. Weber and H.J. Neusser, J. Chem. Phys. 91 (1989) 4555.

[12] U. Schubert, E. Riedle, H.J. Neusser and E.W. Schlag, Israel J. Chem. 30 (1990) 197.

[13] E. Riedle, Th. Weber, U. Schubert, H.J. Neusser and E.W. Schlag, J. Chem. Phys. 93 (1990) 967.

[14] E. Riedle, H.J. Neusser and E.W. Schlag, Phil. Trans. Roy. Soc. London A 332 (1990) 189

[ 15 ] J.H. Callomon, T.M. Dunn and I.M. Mills, Phil. Trans. Roy. Soc. London Ser. A 259 (1966) 499.

[16] G.II. Atkinson and C.S. Parmenter, J. Mol. Spectry. 73 (1978) 31 .

[17] T.A. Stephenson, P.L. Radloff and S.A. Rice, J. Chem. Phys. 81 (1984) 1060 .

[18] T.A. Stephenson and S.A. Rice, J. Chem. Phys. 81 (1984) 1073.

[19] R.L. Rosman, A.A. Villaeys, K.F. Freed and S.A. Rice, J. Chem. Phys. 86 ( 1987 ) 2576.

[20] Th. Weber, A. von Bargen, E. Riedle and H.J. Neusser, J. Chem. Phys. 92 (1990) 90.

[21] S. Gerstenkorn and P. Luc, Atlas du Spectre d'Absorption de la Molecule de l'Iode (CNRS, Paris, 1978).

[22] S. Gerstenkorn and P. Luc, Rev. Phys. Appl. 14 (1979) 791. 
[23] P.D. Willson and S.R. Polo, J. Opt. Soc. Am. 71 (1981) 599.

[24] J.J. Snyder, Appl. Optics 19 (1980) 1223.

[25] J. Pliva and C.A. Martin, J. Mol. Spectry. 91 (1982) 218.

[26] M. Oldani, R. Widmer, G. Grassi and A. Bauder, J. Mol. Struct. 190 (1988) 31.

[27] E.B. Wilson Jr., J.C. Decius and P.C. Cross, Molecular Vibrations (McGraw-Hill, New York, 1955).
[28] G. Herzberg, Infrared and Raman Spectra of Polyatomic Molecules (Van Nostrand, New York, 1945).

[29] A.R. Hoy and I.M. Mills, J. Mol. Spectry. 46 (1973) 333.

[30] G. Amat, H.H. Nielsen and G. Tarrago, Rotation-Vibration of Polyatomic Molecules (Dekker, New York, 1971).

[31] M.-F. Jagod and T. Oka, J. Mol. Spectry. 139 (1990) 313.

[32] A.A. Villaeys and K.F. Freed, Chem. Phys. Letters 123 (1986) 515. 